\title{
Chitosan nanoparticles containing Physalis alkekengi-L extract: preparation, optimization and their antioxidant activity
}

\author{
REZA MAHMOUDI ${ }^{1}$, MARYAM TAJALI ARDAKANI ${ }^{1}$, BEHNAM HAJIPOUR VERDOM ${ }^{2}$, \\ ABOUZAR BAGHERI ${ }^{3}$, HOSSEIN MOHAMMAD-BEIGI ${ }^{4}$, FARHANG ALIAKBARI ${ }^{5,6}$, \\ ZEINAB SALEHPOUR ${ }^{7}$, MOHSEN ALIPOUR ${ }^{8}$, SAJAD AFROUZ ${ }^{1}$ and HASSAN BARDANIA ${ }^{1, *}$ (D) \\ ${ }^{1}$ Cellular and Molecular Research Center, Yasuj University of Medical Sciences, Yasuj 7591994799, Iran \\ ${ }^{2}$ Department of Biophysics, Faculty of Biological Sciences, Tarbiat Modares University, Tehran 14115-111, Iran \\ ${ }^{3}$ Department of Clinical Biochemistry and Genetics, Faculty of Medicine, Mazandaran University of Medical Sciences, \\ Sari 48471-91971, Iran \\ ${ }^{4}$ Interdisciplinary Nanoscience Centre (iNANO) and Department of Molecular Biology and Genetics, Aarhus University, \\ Gustav Wieds Vej 14, DK-8000 Aarhus C, Denmark \\ ${ }^{5}$ Bioprocess Engineering Department, Institute of Industrial and Environmental Biotechnology, National Institute of \\ Genetic Engineering and Biotechnology, Tehran 1497716316, Iran \\ ${ }^{6}$ Department and Center for Biotechnology Research, School of Medicine, Semnan 35198-99951, Iran \\ ${ }^{7}$ Medicinal Plant Research Center, Yasuj University of Medical Sciences, Yasuj 7591994799, Iran \\ ${ }^{8}$ Department of Advanced Medical Sciences and Technologies, School of Medicine, Jahrom University of Medical \\ Sciences, Jahrom 7414846199, Iran \\ *Author for correspondence (hasan.bardania@yums.ac.ir)
}

MS received 12 July 2018; accepted 27 November 2018; published online 25 April 2019

\begin{abstract}
Physalis alkekengi- $L$ is a medicinal herb with a high antioxidant capacity. It is used to treat various diseases. In this study, encapsulation of the hydro-alcoholic extract of $P$. alkekengi-L into chitosan nanoparticles (CNPs) was optimized by using response surface methodology, and its antioxidant capacity and anticancer activity were investigated. The results of the antioxidant capacity evaluation showed that the antioxidant activity of the chitosan nanoparticles containing extract did not significantly decrease by increasing the time of storage (over a 12 day period) as compared to that of un-encapsulated extracts. In conclusion, CNPs can be used to encapsulate and improve the stability of $P$. alkekengi-L extract and its antioxidant properties.
\end{abstract}

Keywords. Physalis alkekengi-L; chitosan nanoparticles; antioxidant activity; stability of extract.

\section{Introduction}

Physalis alkekengi- $L$ is a member of the Solanaceae family, widely grown in Europe and Asia (especially in the northeastern part of China) [1]. This herbal plant has sweet fruits with slight acidity, which are traditionally used as herbal medicine [2]. The fruit extract of $P$. alkekengi-L contains many valuable metabolites including physalins [3,4], carotenoids [5,6], alkaloids and flavonoids [7,8]. It has recently been reported that the fruit extract of $P$. alkekengi-L has many pharmacological properties [1], such as anti-inflammatory $[9,10]$, anticancer [11,12], antioxidant [13], hypoglycemic and analgesic effects $[14,15]$.

In a previous study, Wu et al [13] showed that the ethanol extract of P. peruviana- $L$ (another species of Physalis) has potent antioxidant activity. The high antioxidant capacity of
Physalis extracts is associated with a high level of polyphenol compounds (like ascorbic acid) and flavonoids [16]. The level of ascorbic acid in Physalis fruits ( $46 \mathrm{mg}$ per $100 \mathrm{~g}$ ) is higher than that in other fruits such as pear (4 mg per $100 \mathrm{~g}$ ), apple (6 mg per $100 \mathrm{~g}$ ) and peach ( $7 \mathrm{mg}$ per $100 \mathrm{~g}$ ), and is somewhat comparable to that of orange (50 $\mathrm{mg}$ per $100 \mathrm{~g}$ ) and strawberry $(60 \mathrm{mg}$ per $100 \mathrm{~g})[16,17]$. Additionally, Ramadan and Joerg [18] revealed that there are high levels of antioxidants, water and fat-soluble bioactive materials in the juice of $P$. peruviana- $L$, comparable to other products commonly available in the market. Moreover, it has been reported that $P$. peruviana fruit extracts have anticancer activity and immune-modulatory potential for the development of new complementary pharmaceutical products [19].

Bioactive components like phenolic compounds with high antioxidant activity are unstable in the oxidative environment, 
which can limit the application of these compounds [20]. Therefore, we need to use some methods to protect bioactive materials from oxygen, water, pH changes, light, etc. Encapsulation technology can be used to enclose bioactive materials into a polymer matrix, and increase their shelf life and efficacy by protecting them.

Chitosan, a natural polysaccharide derivative of chitin, is a poly-cationic linear polymer that has high potential to encapsulate natural ingredients. Chitosan has some unique properties. It is biocompatible, non-toxic and biodegradable, and has good bio-adhesive properties [21], making it capable of in vivo use through oral [22], intraperitoneal [23] or intravenous administrations [24]. Additionally, chitosan nanoparticles (CNPs) can be easily prepared based on ionic gelation between positively charged chitosan polymer tripolyphosphate (TPP) anions [21]. Therefore, it seems that CNPs have high capacity for delivering natural extracts of medicinal plants like $P$. alkekengi-L.

In this study, we developed CNPs containing P. alkekengi$L$ extract and characterized them using scanning electron microscopy (SEM) and dynamic light scattering (DLS). The optimal conditions for extract encapsulation into CNPs were explored using a central composite design (CCD) combined with response surface methodology (RSM). The CCD was developed through Design Expert Software (DX-7, StateEase Inc., Version 7.0.0) to investigate the effects of stirring time and chitosan, TPP and extract concentrations on the encapsulation efficiency (EE) of hydro-alcoholic extract into CNPs. Thereafter, the antioxidant capacity of the CNP-CE was evaluated using 2,2-diphenylpicrylhydrazyl (DPPH) and ferric reducing antioxidant power (FRAP) assays.

\section{Materials and methods}

\subsection{Materials}

DPPH, 2,4,6-tri(2-pyridyl)-s-triazine (TPTZ), 3-(4,5-dimethylthiazol-2-yl)-2,5-diphenyl tetrazolium bromide, chitosan (low molecular weight) and sodium TPP were purchased from Sigma (USA) and ferric chloride hexahydrate $\left(\mathrm{FeCl}_{3} \cdot 6 \mathrm{H}_{2} \mathrm{O}\right)$, ferrous chloride tetrahydrate $\left(\mathrm{FeCl}_{2} \cdot 4 \mathrm{H}_{2} \mathrm{O}\right)$, aluminium chloride $\left(\mathrm{AlCl}_{3}\right)$, sodium nitrite $\left(\mathrm{NaNO}_{2}\right)$ and methanol from Merck (Germany). Dulbecco's modified eagle's medium, foetal bovine serum, trypsin and pen/strep solution were from GibcoBRL (Life Technologies, Paisley, Scotland). Annexin V-FITC and propidiumiodide kit were prepared from eBiosciences (San Diego, CA, USA). The human colon adenocarcinoma cell line (HT29) was obtained from the Pasteur Institute of Iran.

\subsection{P. alkekengi-L extract}

The plant seeds of $P$. alkekengi- $L$ were collected from Kohgiluyeh and Boyer-Ahmad province, Iran. The seeds were dried in the dark at room temperature and powdered by using an electric grinder. The extraction of a known amount of the sample was prepared with $80 \%$ methanol using the percolation method for $72 \mathrm{~h}$. The resulting extract was then concentrated by using a rotary evaporator (Heidolph, Germany) and dried using a freeze-dryer (Christ freeze-dryer, ALPHA 2-4 LD plus).

\subsection{Synthesis of CNP-CE}

CNPs containing $P$. alkekengi- $L$ extract were synthesized by using the ionic gelation method [25]. Briefly, a desired amount of chitosan dissolved in acetic acid (1\%) was mixed with a certain amount of extract under magnetic stirring (1000 rpm) at room temperature. After 5 min of stirring, TPP solution was added dropwise to the mixture to form nanoparticles. Various amounts of chitosan, extract and TPP were used according to the designed experiment (supplementary table S1). The solution of the prepared nanoparticles was centrifuged at 10000 $\mathrm{rpm}$ for $10 \mathrm{~min}$. The supernatants were used to determine the amount of extract encapsulated into the nanoparticles.

\section{$2.4 \quad E E$}

The percentage of the encapsulated extract into CNPs was determined by using a total flavonoid assay according to the following equation.

$$
\begin{aligned}
& \text { EE }(\%)=\text { Actual flavonoid of } P . \text { alkekengi- } L \text { extract } \\
& \text { entrapped in nanoparticles/Total flavonoid of initial } \\
& \text { extract } \times 100 \text {. }
\end{aligned}
$$

The total flavonoid content of the samples was determined by the $\mathrm{UV}$ absorption of the $\mathrm{AlCl}_{3}$-flavonoid complex based on standard rutin [26]. For this, $0.5 \mathrm{ml}$ of sample solution and $150 \mu \mathrm{l}$ of $5 \% \mathrm{NaNO}_{2}$ were mixed with $2 \mathrm{ml}$ of distilled water and incubated for $6 \mathrm{~min}$ at room temperature. Then, $150 \mu \mathrm{l}$ of $10 \% \mathrm{AlCl}_{3}$ solution was added and again after 6 min of incubation, $2 \mathrm{ml}$ of $4 \% \mathrm{NaOH}$ was added to the mixture. The absorbance of the samples was recorded by using a spectrophotometer at $415 \mathrm{~nm}$ and the standard curve was prepared using different concentrations of rutin solutions. The total flavonoid content was expressed as rutin equivalents (mg rutin per g sample) through the calibration curve of rutin.

\subsection{Optimization of $C N P-C E$}

To optimize the EE of extraction into CNPs, a fourth-order experimental design and mathematical model are required. In the present study, four independent variables (stirring time (min) and the concentration of chitosan (mg), TPP (mg) and extract $\left(\mathrm{mg} \mathrm{ml}^{-1}\right)$ ) were selected based on the preliminary experiments and the literature survey. RSM analysis was performed by using a CCD involving 30 experiments of four variables at five levels $(-2,-1,0,+1$ and +2$)$ applying full factorial and six centre points to optimize the CNP-CE (supplementary table S1). The ranges and levels of the examined 
independent variables are presented in supplementary data (supplementary table S2). The modified quadratic model for predicting the optimum condition is presented in the following equation:

$$
y=\beta_{0}+\sum_{i} \beta_{i} X_{i}+\sum_{i} \beta_{i i} X_{i}^{2}+\sum_{i \neq j} \beta_{i j} X_{i} X_{j},
$$

where $y$ is the expected response (EE\%) and $X_{i}$ and $X_{j}$ are independent variables. In addition, $\beta_{0}, \beta_{i}, \beta_{i i}$ and $\beta_{i j}$ are constant term, linear coefficient, quadratic coefficient and interactive term coefficient, respectively.

\subsection{Characterization of $C N P-C E$}

The morphology and size of the CNP-CE were evaluated using SEM (KYKY-EM3200, KYKY Technology Development Ltd., Beijing, China). The hydrodynamic particle size and zeta potential of nanoparticles were also determined by using a Zetasizer Nano ZS instrument (Malvern Instruments Ltd, Malvern, Worcestershire, UK).

\subsection{Antioxidant assays}

Two antioxidant assays (DPPH and FRAP) were carried out to evaluate the stability and antioxidant capacity of the extract at pre-determined periods of time. For this, un-encapsulated and extracts encapsulated into nanoparticles were stored at room temperature for 12 days, and their antioxidant capacity was investigated.

2.7a DPPH assay: The DPPH radical scavenging assay was performed according to the previously reported method [27]. Briefly, $0.5 \mathrm{ml}$ of samples $\left(4 \mathrm{mg} \mathrm{ml}^{-1}\right.$ of extract and certain amounts of nanoparticles containing $4 \mathrm{mg} \mathrm{ml}^{-1}$ of extract) was added to $1 \mathrm{ml}$ of $0.1 \mathrm{mM} \mathrm{DPPH}^{-}$methanolic solution and vigorously shaken in the dark at room temperature. The absorbance of samples at $517 \mathrm{~nm}$ was measured after $30 \mathrm{~min}$. Ascorbic acid and methanol were used as positive (standard) and negative controls, respectively. The antioxidant capacity of empty CNPs was also assessed at the concentration equivalent to those of the CNP-CE. The antioxidant capacity as percentage effect (E\%) was calculated using the following equation:

Percentage effect $(\mathrm{E} \%)=[($ Abs control - Abs sample $)$ /Abs control] $\times 100$.

2.7b FRAP assay: FRAP assay as another antioxidant capacity assay was used to evaluate the ferric reducing activity of samples. This procedure was carried out according to the previously described methods [28]. Briefly, $2.5 \mathrm{ml}$ of $10 \mathrm{mM}$ TPTZ and $2.5 \mathrm{ml}$ of $20 \mathrm{mM} \mathrm{FeCl}_{3}$ were added to $0.25 \mathrm{mM}$ of acetate buffer, at $\mathrm{pH} 3.6$ to prepare a fresh FRAP reagent. Three hundred microlitres of samples $\left(4 \mathrm{mg} \mathrm{ml}^{-1}\right.$ of extract and certain amount of nanoparticles containing $4 \mathrm{mg} \mathrm{ml}^{-1}$ ) was mixed with $2 \mathrm{ml}$ of FRAP reagent and incubated at room temperature for $10 \mathrm{~min}$. The absorbance of the samples was spectrophotometrically read at $593 \mathrm{~nm}$. Acetate buffer and $1 \mathrm{mM} \mathrm{Fe}(\mathrm{II})\left(\mathrm{FeSO}_{4} \cdot 7 \mathrm{H}_{2} \mathrm{O}\right)$ were used as positive (standard) and negative controls, respectively. The antioxidant capacity of empty CNPs was also assessed at the concentrations equivalent to those of the CNP-CE. The standard curve was prepared by using several concentrations of $\mathrm{Fe}$ (II) $\left(\mathrm{FeSO}_{4} \cdot 7 \mathrm{H}_{2} \mathrm{O}\right)$ between 100 and $500 \mu \mathrm{g}(100,200,300,400$ and $500 \mu \mathrm{g}$ ).

\subsection{Statistical analysis}

All experiments were performed three times, and the data were expressed as the mean \pm standard deviation (SD). The difference between groups was analysed by the $t$-test and one-way ANOVA. $p<0.05$ was considered statistically significant.

\section{Results and discussion}

\subsection{Optimization of encapsulation of extraction into nanoparticles}

The effect of four factors (stirring time and concentrations of chitosan, TPP and extract) on the EE of extraction into nanoparticles was examined using 30 experimental runs generated by the CCD. The results of ANOVA are presented in table 1 ; the results show that $R^{2}$ and adj- $R^{2}$ have high values indicating that the model is highly significant and appropriate $\left(p<0.05, R^{2}=0.9753\right.$ and adj- $\left.R^{2}=0.9623\right)$. The results show a satisfactory correlation between the experimental data and predicted values as a result of the clustering of points around the diagonal line, confirming the robustness of the model (supplementary figure S1). The best acquired conditions for the encapsulation of extraction into CNPs include $41.78 \mathrm{~min}$ stirring time, $4.75 \mathrm{mg} \mathrm{ml}^{-1}$ extraction, $5 \mathrm{mg}$ chitosan and $3.11 \mathrm{mg}$ TPP. According to the optimum conditions, three experiments were conducted to validate the model. The EE of the extract into CNPs in a lab experiment was $95 \pm 3.2$, well within the allowed experimental error (supplementary table S3). The optimization of extract entrapment in CNPs by RSM indicated a significant correlation between independent variables. The encapsulation of the extract into CNPs ascended when the two parameters (extract and chitosan concentration) increased. However, the stirring time and TPP concentration did not have a significant positive effect on the EE. A high EE was achieved with a moderate TPP concentration and stirring time, and high concentrations of chitosan and extract (supplementary figure S2). The EE of the extract increased with an increase in the amount of chitosan and extract. Hydro-alcoholic extract contains many polyphenol compounds, which have a high affinity to interact with positive polymers (chitosan), resulting in a 
Table 1. ANOVA test for response surface reduced quadratic model.

\begin{tabular}{|c|c|c|c|c|c|c|}
\hline Source & & Sum of squares & $\mathrm{DF}$ & Mean squares & $F$ value & $P$ value \\
\hline Model & & 24448.94081 & 10 & 2444.894081 & 75.1171 & $<0.0001$ \\
\hline A - Extraction concentration & & 23958.21769 & 1 & 23958.21769 & 736.0938 & $<0.0001$ \\
\hline B - Stirring time & & 18.38970882 & 1 & 18.38970882 & 0.5650 & 0.4615 \\
\hline $\mathrm{C}-\mathrm{TPP}$ concentration & & 70.64604383 & 1 & 70.64604383 & 2.1705 & 0.1571 \\
\hline D - Chitosan concentration & & 160.2536956 & 1 & 160.2536956 & 4.9236 & 0.0389 \\
\hline $\mathrm{AB}$ & & 14.29983758 & 1 & 14.29983758 & 0.4393 & 0.5154 \\
\hline $\mathrm{AD}$ & & 93.3902973 & 1 & 93.3902973 & 2.8693 & 0.1066 \\
\hline $\mathrm{BD}$ & & 21.36148577 & 1 & 21.36148577 & 0.6563 & 0.4279 \\
\hline $\mathrm{CD}$ & & 63.7313749 & 1 & 63.7313749 & 1.9581 & 0.1778 \\
\hline $\mathrm{C}^{2}$ & & 21.24522236 & 1 & 21.24522236 & 0.6527 & 0.4291 \\
\hline $\mathrm{D}^{2}$ & & 22.00048609 & 1 & 22.00048609 & 0.6759 & 0.4212 \\
\hline Residual & & 618.4078108 & 19 & 32.54777952 & & \\
\hline Lack of fit & & 573.3109367 & 14 & 40.9507812 & 4.5403 & 0.0522 \\
\hline$R$-squared & 0.9753 & & & & & \\
\hline Adj. $R$-squared & 0.9623 & & & & & \\
\hline
\end{tabular}
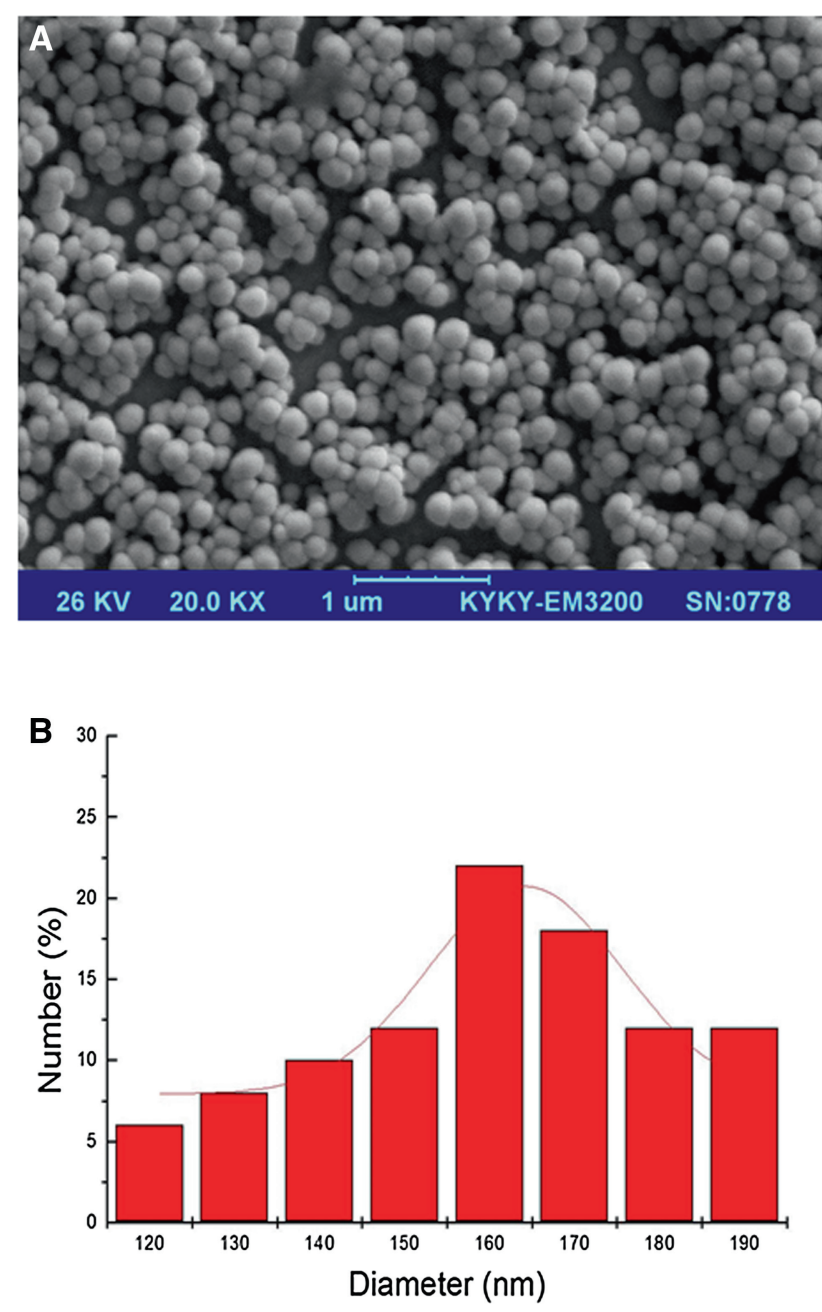

Figure 1. (A) SEM images and (B) histogram particle size distribution of CNP-CEs.
Table 2. Size and $\zeta$ potential measurement of empty CNPs and CNP-CEs.

\begin{tabular}{lcc}
\hline Sample & Size $($ d. $n m)$ & Zeta potential $(\mathrm{mV})$ \\
\hline CNP-CE & $196 \pm 20$ & $7.69 \pm 5.7$ \\
CNP & $188.9 \pm 15$ & $25 / 3 \pm 7 / 19$ \\
\hline
\end{tabular}

higher entrapment of extraction into CNPs [29]. Additionally, Feng et al [30] showed that the encapsulation of drugs into CNPs is associated with the chitosan concentration.

\subsection{Characterization of $C N P-C E$}

SEM and DLS analyses were utilized for the characterization of the CNP-CE. The results of SEM revealed that CNP-CEs have a nearly uniform size of $\sim 167 \pm 25 \mathrm{~nm}$ in diameter (figure 1). The hydrodynamic size of nanoparticles measured by DLS analysis was bigger than that obtained by SEM (table 2). The bigger hydrodynamic size may be due to the high swelling capacity of CNPs [30].

The zeta potential of the CNP-CE was measured using DLS analysis; the results show that the zeta potential of the CNP$\mathrm{CE}$ is lower than that of empty CNPs (table 2). This may be attributed to the loading of nanoparticles which contain several polyphenol compounds and neutralize the cationic charge of CNPs. These results are in accordance with previous results reported by Han et al [31].

\subsection{Stability of CNP-CE}

The stability of the CNP-CE was evaluated by assessing its antioxidant activity using FRAP and DPPH assays in pre-determined periods of time. For this, the un-encapsulated 

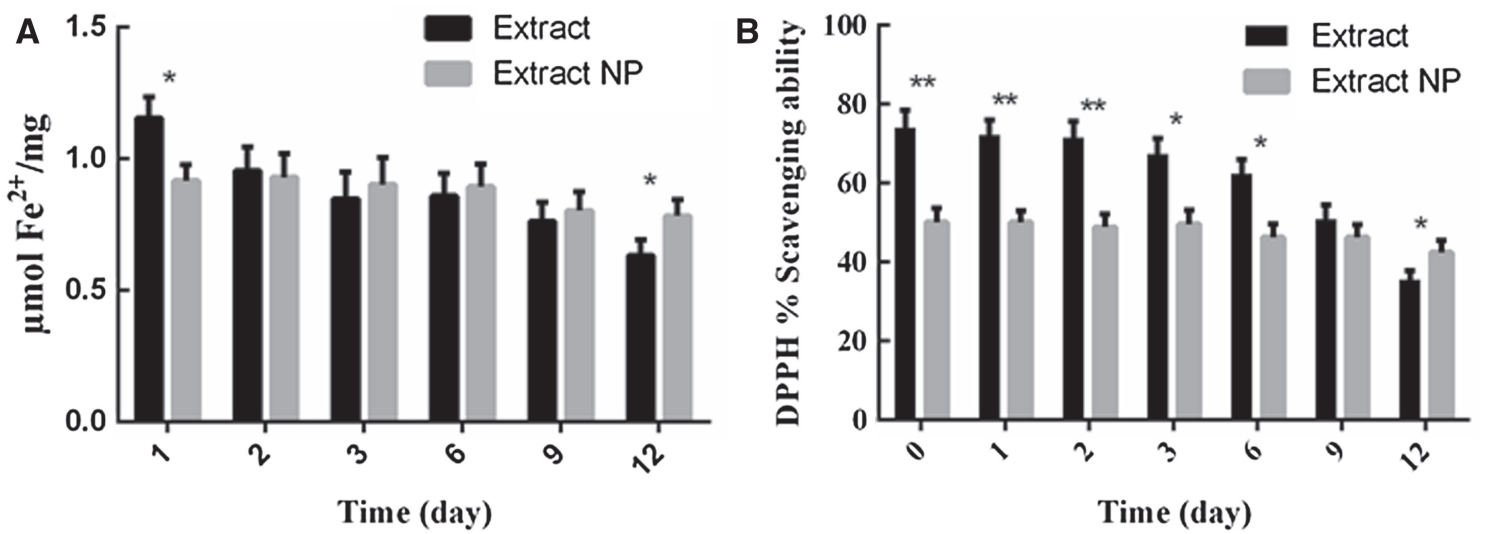

Figure 2. The antioxidant (A) FRAP and (B) DPPH radical scavenging capacity of the un-encapsulated extract and CNP-CE during storage. Data are expressed as mean $\pm \mathrm{SD}$ of three independent experiments. $*$ and $* *$ denote a statistically significant $(p<0.05$ and $p<0.01)$ difference from the untreated control.

extract and CNP-CE were stored at room temperature for 12 days, and their antioxidant activity was evaluated. Figure 2 shows that by increasing the time of storage, the antioxidant capacity of the un-encapsulated extract decreased significantly. However, the decrease in the antioxidant capacity of the CNP-CE was not significant. In the initial days (according to both antioxidant assessing methods), the antioxidant capacity of the un-encapsulated extract is significantly more than that of the extract encapsulated into CNPs; but after 12 days, the antioxidant capacity of the un-encapsulated extract significantly decreased as compared to that of the CNP-CE. The extract encapsulated into the nanoparticles was not exposed to destructive factors including oxygen, water, $\mathrm{pH}$ changes and light; and was slowly released from the nanoparticles. Therefore, its stability is more than that of the un-encapsulated extract. These results are in line with the previous reports of Han et al [32], Tang et al [33], and Jeon et al. [34]. The stability of protease encapsulated into CNPs was evaluated by Tang et al [33] and they showed that the stability of protease encapsulated into CNPs increased when exposed to temperature and pH. Additionally, Han et al [32] encapsulated the extract of jujube seeds and pulp into CNPs and evaluated the stability of the extract via antioxidant assays. They revealed that the antioxidant activity of the extract encapsulated into CNPs did not change significantly after 12 days, while the antioxidant capacity of the un-encapsulated extract decreased significantly. Moreover, Jeon et al [34] evaluated the stability and solubility of resveratrol encapsulated into CNPs, and showed that resveratrol encapsulated into CNPs tends to significantly maintain its solubility and antioxidant activity after 15 days of storage as compared with the non-encapsulated resveratrol.

\section{Conclusions}

CNPs containing $P$. alkekengi- $L$ extract were successfully optimized using RSM. The optimal conditions to achieve the maximum EE of extract into nanoparticles $(95 \pm 3.2 \%)$ are
$4.75 \mathrm{mg} \mathrm{ml}^{-1}$ extract, $41.78 \mathrm{~min}$ stirring time, $5 \mathrm{mg}$ chitosan and $3.11 \mathrm{mg}$ TPP. The encapsulation of $P$. alkekengi- $L$ extract into CNPs effectively improved the antioxidant capacity of the extract by protecting the extract bioactive substances like flavonoids and increasing their stability. These results demonstrated that the encapsulation of $P$. alkekengi- $L$ extracts into CNPs can effectively improve the antioxidant activity of the $P$. alkekengi- $L$ extract.

\section{Acknowledgements}

This work was financially supported by the Yasuj University of Medical Sciences.

\section{References}

[1] Li X, Zhao J, Yang M, Liu Y, Li Z, Li R et al 2014 Phytochem. Lett. 1095

[2] Ge Y, Duan Y, Fang G, Zhang Y and Wang S 2009 J. Sci. Food Agric. 891593

[3] Zheng Y, Luan L, Chen Y, Ren Y and Wu Y 2012 J. Pharm. Biomed. Anal. 7154

[4] Huang C, Xu Q, Chen C, Song C, Xu Y, Xiang Y et al 2014 J. Chromatogr. A 1361139

[5] Deineka V, Sorokopudov V, Deineka L, Tret'yakov M Y and Fesenko V 2008 Pharm. Chem. J. 4287

[6] Pintea A, Varga A, Stepnowski P, Socaciu C, Culea M and Diehl H A 2005 Phytochem. Anal. 16188

[7] Basey K and Jack G W 1973 Phytochemistry 122557

[8] Kranjc E, Albreht A, Vovk I, Makuc D and Plavec J 2016 J. Chromatogr. A 143795

[9] Bastos G, Silveira A, Salgado C, Picanço-Diniz D and Do Nascimento J 2008 J. Ethnopharmacol. 118246

[10] Vieira A T, Pinho V, Lepsch L B, Scavone C, Ribeiro I M, Tomassini T et al 2005 Br. J. Pharmacol. 146244

[11] Magalhães H I F, Torres M R, Costa-Lotufo L V, Moraes M O, Pessoa C, Veras M L et al 2006 J. Pharm. Pharmacol. 58235 
[12] Makino B, Ohya J, Yamamura H, Araki S, Butsugan Y and Kawai M 2003 Pharmazie 5870

[13] Wu S, Tsai J, Chang S, Lin D, Wang S, Huang S et al 2006 J. Ethnopharmacol. 108407

[14] Wang H P, Ms X V and Sun L 2004 J. Tradit. Chin. Med. 130

[15] Gong S, Shan L D and Zhang Y Y 2002 Acta Academiae Medicinae Suzhou 410

[16] Mohamed F R 2011 Food Res. Int. 441830

[17] Belitz H and Grosch W 1999 Food chemistry (Berlin: SpringerVerlag)

[18] Ramadan M F and Joerg T M 2007 J. Sci. Food Agric. 87 452

[19] Mier-Giraldo H, Díaz-Barrera L E, Delgado-Murcia L G, Valero-Valdivieso M F and Cáez-Ramírez G 2017 J. Evid. Based Complement. Altern. Med. 22777

[20] Woranuch S and Rangrong Y 2013 Carbohydr. Polym. 96 578

[21] Agnihotri S A, Mallikarjuna N N and Aminabhavi T M 2004 J. Control. Release $\mathbf{1 0 0} 5$

[22] Servat-Medina L, González-Gómez A, Reyes-Ortega F, Sousa I M O, Queiroz N D C A, Zago P M W et al 2015 Int. J. Nanomed. 103897
[23] Howard K A 2009 Adv. Drug Deliv. Rev. 61710

[24] Hee-Dong H, Mangala L S, Lee J W, Shahzad M M, Kim H S, Shen D Y et al 2010 Clin. Cancer Res. 163910

[25] Lee J S, Kim G H and Lee H G 2010 J. Agric. Food Chem. 58 3316

[26] Karimian P, Kavoosi G and Amirghofran Z 2013 J. Immunotoxicol. 10393

[27] Chen Z, Bertin R and Froldi G 2013 Food Chem. 138414

[28] Griffin Sean P and Ranjeet B 2004 J. Exp. Mar. Bio. Ecol. 302 201

[29] Liang J, Li F, Fang Y, Yang W, An X, Zhao L et al 2010 Eur. Food Res. Technol. 231917

[30] Feng C, Wang Z, Jiang C, Kong M, Zhou X, Li Y et al 2013 Int. J. Pharm. 457158

[31] Li L, Chen D, Zhang Y, Deng Z, Ren X, Meng X et al 2007 Nanotechnology 18405102

[32] Han H J, Lee J S, Park S A, Ahn J B and Lee H G 2015 Colloids Surf. B: Biointerfaces 13093

[33] Tang Z X, Qian J Q and Shi L E 2006 Process Biochem. 41 1193

[34] Jeon Y O, Lee J S and Lee H G 2016 Colloids Surf. B: Biointerfaces 147224 